\title{
THE CLOSED LEAF INDEX OF FOLIATED MANIFOLDS
} BY

\author{
LAWRENCE CONLON AND SUE GOODMAN
}

\begin{abstract}
For $M$ a closed, connected, oriented 3-manifold, a topological invariant is computed from the cohomology ring $H^{*}(M ; \mathrm{Z})$ that provides an upper bound to the number of topologically distinct types of closed leaves any smooth transversely oriented foliation of $M$ can contain. In general, this upper bound is best possible.
\end{abstract}

Introduction. Let $M$ be a closed, connected, oriented $n$-manifold, $F$ a smooth transversely oriented foliation of codimension one on $M$. Throughout this paper, the term "foliated $n$-manifold" will mean such a pair $(M, F)$.

Definition. The closed leaf index of $F$ is the number $\gamma(F)$ of distinct topological types of closed leaves in $F$.

It is easy to see that $\gamma(F)$ is finite. By a theorem of S. P. Novikov [3], all foliations of $S^{3}$ have $\gamma(F)=1$, and those of $S^{1} \times S^{2}$ have $\gamma(F)>1$. It is also known [2] that foliations of $T^{3}$ satisfy $0<\gamma(F)<2$ and that those of $S^{1} \times S^{2}$ satisfy $1<\gamma(F)<2$, each of these possibilities being realized by a suitable foliation. By [1], the greatest lower bound of $\gamma(F)$ on any closed 3-manifold $M$ is always 0 or 1 . It is a result of P. Schweitzer [5] that, for $n>5$, every $n$-manifold that can be foliated admits a $C^{0}$ foliation $F$ (with $C^{\infty}$ leaves) having $\gamma(F)=0$. In this paper we will produce a fairly severe upper bound to the closed leaf index of foliated 3-manifolds. Analogous but weaker results will be obtained in higher dimensions.

Definition. The symbol $\alpha(M)$ denotes the largest integer such that some basis $x_{1}, \ldots, x_{r}$ of $H^{1}(M ; Z)=Z^{r}$ satisfies $x_{i} \cup x_{j}=0,1<i, j<\alpha(M)$.

For instance, $\alpha\left(S^{3}\right)=0, \alpha\left(S^{1} \times S^{2}\right)=1$, and $\alpha\left(T^{3}\right)=1$. Indeed, we will compute $\alpha$ for all oriented $S^{1}$-bundles over closed oriented surfaces (\$3).

It is convenient to define a strictly increasing function $\varphi: \mathbf{Z}^{+} \rightarrow \mathbf{Z}^{+}$by

$$
\varphi(k)= \begin{cases}k+1, & k=0,1, \\ 3 k-2, & k \geqslant 2 .\end{cases}
$$

Theorem A. If $(M, F)$ is a foliated 3-manifold, then $\gamma(F)<\varphi(\alpha(M))$.

THEOREM B. For each integer $k \geq 0$, there is a foliated 3-manifold $\left(M_{k}, F_{k}\right)$ such that $\alpha\left(M_{k}\right)=k$ and $\gamma\left(F_{k}\right)=\varphi(k)$.

Received by the editors May 14, 1975 and, in revised form, March 29, 1976.

AMS (MOS) subject classifications (1970). Primary 57D30; Secondary 57D95.

- American Mathematical Society 1977 
We do not know, however, whether Theorem $\mathrm{A}$ gives the best possible upper bound for $\gamma(F)$ on every 3-manifold. We conjecture that this fails, in fact, for the "least twisted" nontrivial oriented $S^{1}$-bundle over a closed, oriented surface of genus $g>0$.

In higher dimensions it is not reasonable to expect such sharp information on $\gamma(F)$, but the following invariants are manageable.

Definition. The homology index of $F$ is the number $h(F)$ of classes in $H_{n-1}\left(M^{n} ; \mathbf{Z}\right)$, distinct modulo sign, that can be represented by closed leaves of $F$. The mod 2 homology index $h_{2}(F)$ is the number of distinct classes in $H_{n-1}\left(M^{n} ; \mathbf{Z}_{2}\right)$ so represented.

Definition. The Euler index of $F$ is the number $e(F)$ of distinct integers $|\chi(L)|$ for closed leaves $L$ of $F$.

On even dimensional manifolds, the Euler index is always 0 or 1 ; hence this invariant is of real significance only on odd dimensional manifolds. For foliated 3-manifolds, the equality $\gamma(F)=e(F)$ is immediate from the classification of closed surfaces and the Reeb stability theorem, so Theorem $A$ and Theorem $B$ are specializations respectively of Theorem $A^{\prime}$ and Theorem B' $^{\prime}$ below.

THEOREM $\mathrm{A}^{\prime}$. If $(M, F)$ is a foliated n-manifold, then $e(F)<h(F)=h_{2}(F)$ $<\varphi(\alpha(M))$.

THEOREM $\mathrm{B}^{\prime}$. For each pair of integers $(n, k) \neq(4,0)$ with $n>3$ and $k>0$, there is a foliated $n$-manifold $\left(M_{k}^{n}, F_{k}\right)$ with $\alpha\left(M_{k}^{n}\right)=k$ and $h\left(F_{k}\right)=\varphi(k)$. If $n$ is odd, then also $\gamma\left(F_{k}\right)>e\left(F_{k}\right)=\varphi(k)$.

The nonexistence of $\left(M_{0}^{4}, F_{0}\right)$ is seen by noting that $H^{1}\left(M_{0}^{4}\right)=0$. By Poincare duality the relation $\chi\left(M_{0}^{4}\right)>2$ must hold and, consequently, there can be no foliation of $M_{0}^{4}$ of codimension one.

Theorem $\mathrm{A}^{\prime}$ involves a series of topological and combinatorial considerations. Theorem $\mathrm{B}^{\prime}$ is a consequence of recent deep results of $\mathrm{W}$. Thurston [6]. The authors are grateful to Thurston for preprints of his work.

1. Submanifold systems of codimension one. Let $M$ be a closed, connected, oriented $n$-manifold. A set $\mathfrak{L}=\left\{L_{1}, \ldots, L_{r}\right\}$ of disjoint, imbedded, closed, connected, oriented submanifolds of $M$ of codimension one will be called a submanifold system. In this section we assemble a number of facts about such systems, applications being made later to foliations.

We denote by $\left[L_{i}\right]$ the element of $H_{n-1}(M)$ represented by the fundamental cycle of $L_{i}$, and by $[\mathcal{Q}]$ the set $\left\{\left[L_{1}\right], \ldots,\left[L_{r}\right]\right\}$. Here and elsewhere, unless otherwise specified, all homology and cohomology will have integral coefficients. Note that $H_{n-1}(M) \cong H^{1}(M)$ and that this group is free abelian by the universal coefficient theorem. 
For the usual reasons, any two maximal linearly independent subsets of [L] have the same cardinality, namely the rank of $\operatorname{span}_{z}[\mathcal{L}]$. The geometric interpretation of this fact is that any two subsets of $\mathcal{L}$, maximal with respect to the property of not disconnecting $M$, have the same cardinality. We suppose that $\left\{\left[L_{1}\right], \ldots,\left[L_{k}\right]\right\}$ is a maximal linearly independent subset and define $\operatorname{rank}(\mathfrak{L})=k$.

LEMMA 1.1. The set $\left\{\left[L_{1}\right], \ldots,\left[L_{k}\right]\right\}$ spans a direct summand of $H_{n-1}(M)$. An element $x \in H_{n-1}(M)$ which is linearly dependent on $\left\{\left[L_{1}\right], \ldots,\left[L_{k}\right]\right\}$ can be represented by a closed, connected, oriented submanifold $L \subset M$ disjoint from $L_{i}, 1 \leqslant i \leqslant k$, if and only if $x=\varepsilon_{1}\left[L_{1}\right]+\cdots+\varepsilon_{k}\left[L_{k}\right]$ where each $\varepsilon_{i} \in\{0,1$, $-1\}$.

Proof. Since $W=M-\cup_{i=1}^{k} L_{i}$ is an open connected subspace of $M$, we can let $\sigma_{1}, \ldots, \sigma_{k}$ be disjoint smoothly imbedded circles in $M$ transverse to $\cup_{i=1}^{k} L_{i}$ with $\sigma_{i} \cap L_{j}$ a set of $\delta_{i j}$ points, $1 \leqslant i, j \leqslant k$. By suitably orienting these circles we obtain the homology intersection products $\left[\sigma_{i}\right] *\left[L_{j}\right]=\delta_{i j}$. In particular, $\left\{\left[L_{1}\right], \ldots,\left[L_{k}\right]\right\}$ spans a direct summand of $H_{n-1}(M)$.

If $L \subset W$ is a closed, connected, oriented submanifold of $M$ which does not disconnect $W$, then the above argument shows that $[L]$ is linearly independent of $\left\{\left[L_{1}\right], \ldots,\left[L_{k}\right]\right\}$. But if $L$ does disconnect $W$, then it is clear that $[L]=\sum_{i=1}^{k} \varepsilon_{i}\left[L_{i}\right]$, where each $\varepsilon_{i} \in\{0,1,-1\}$.

If $x=\varepsilon_{1}\left[L_{1}\right]+\cdots+\varepsilon_{k}\left[L_{k}\right]$ and each $\varepsilon_{i} \in\{0,1,-1\}$, we can suppose

$$
x=\left[L_{1}\right]+\cdots+\left[L_{h}\right]-\left[L_{h+1}\right]-\cdots-\left[L_{h+q}\right]
$$

(where either $h$ or $q$ may be 0 ). Let $L_{i}^{\prime}$ be obtained by displacing $L_{i}$ a distance $\varepsilon>0$ along the positive normal trajectories to $L_{i}, 1 \leqslant i<h$, and along the negative normal trajectories if $h+1<i<h+q$. Using the fact that $W$ is connected, we construct simple arcs $\tau_{i}:[0,1] \rightarrow M$ meeting the following specifications. No $\tau_{i}$ meets an $L_{j}, 1<j \leqslant k$, and the interior of no $\tau_{i}$ meets an $L_{j}^{\prime}, 1<j<h+q$. For $1 \leqslant i \leqslant h-1$, the arc $\tau_{i}$ joins the positive side of $L_{i}^{\prime}$ to the positive side of $L_{i+1}^{\prime}$. The arc $\tau_{h}$ joins the positive side of $L_{h}^{\prime}$ to the negative side of $L_{h+1}^{\prime}$. For $h+1 \leqslant i \leqslant h+q-1$, the arc $\tau_{i}$ joins the negative side of $L_{i}^{\prime}$ to the negative side of $L_{i+1}^{\prime}$. The connected sum of the manifolds $L_{i}^{\prime}$ via small tubes along these arcs is a closed, connected, oriented manifold $L$ meeting no $L_{j}, 1 \leqslant j \leqslant k$, such that $x=[L]$.

In particular, we can now write $\left[L_{j}\right]=\sum_{i=1}^{k} \varepsilon_{j}^{i}\left[L_{i}\right], 1 \leqslant j \leqslant r$, where each $\varepsilon_{j}^{i} \in\{0,1,-1\}$. Let $E_{j}$ denote the coefficient $k$-tuple $\left(\varepsilon_{j}^{1}, \varepsilon_{j}^{2}, \ldots, \varepsilon_{j}^{k}\right)$ of $\left[L_{j}\right]$.

LEMMA 1.2. If $\left\{\left[L_{p}\right],\left[L_{q}\right]\right\}$ is linearly independent, then $E_{p}$ and $E_{q}$ do not have exactly the same zero entries.

Proof. We can assume $p, q>k$. The set consisting of $L_{p}$ together with those $L_{i}$ for which $\varepsilon_{p}^{i} \neq 0$ (say $1 \leqslant i<h \leqslant k$ after suitable renumbering) 
disconnects the manifold into two components, $W_{1}$ and $W_{2}$, with common boundary $L_{p} \cup L_{1} \cup \cdots \cup L_{h}$. The manifold $L_{q}$ lies in one of these components, say $W_{1}$, and if $E_{q}$ has exactly the same zero entries as $E_{p}$, then $L_{q}$ separates $W_{1}$ into two components, one of which has boundary $L_{q} \cup L_{1}$ $\cup \cdots \cup L_{h}$. It follows that the other component has boundary $L_{p} \cup L_{q}$, contradicting the linear independence of $\left\{\left[L_{p}\right],\left[L_{q}\right]\right\}$.

Definition. $\mathcal{L}$ is an admissible system if $[\mathcal{L}]$ is pairwise linearly independent (or, equivalently, if $M-\left(L_{\mathrm{i}} \cup L_{j}\right)$ is connected, $\left.1<i, j<r\right)$.

ReMarK. Denote by $\left[L_{i}\right]_{2}$ the element of $H_{n-1}\left(M ; \mathbf{Z}_{2}\right)$ carried by $L_{i}$ and by $[\mathfrak{L}]_{2}$ the set of these classes. The force of $(1.2)$ is that, for $\mathcal{L}$ admissible, [ $\left.\mathcal{L}\right]$ and $[\mathrm{L}]_{2}$ have the same cardinality.

Let $\mathcal{L}$ be an admissible system. We can modify each $L_{i}$ by attaching small handles $S^{n-2} \times I$ to $L_{i}$ within Euclidean neighborhoods of $M$. If $L_{i}^{\prime}$ denotes the resulting submanifold, then $\left[L_{i}^{\prime}\right]=\left[L_{i}\right]$ and the resulting admissible system $\mathcal{L}^{\prime}$ has exactly the same separation relations in $M$ as did $\mathcal{L}$. Furthermore, if $U_{1}, \ldots, U_{q}$ are the components of $M-\cup L_{i}$ and $U_{1}^{\prime}, \ldots, U_{q}^{\prime}$ the corresponding components of $M-\cup L_{i}^{\prime}$, then each $U_{j}^{\prime}$ is obtained from $U_{j}$ by adjoining and/or cutting out solid handles. If a solid handle was adjoined, then $\chi\left(\bar{U}_{j}^{\prime}\right)=\chi\left(\bar{U}_{j}\right)-1$. If $n$ is even and a solid handle was cut out, then $\chi\left(\bar{U}_{j}^{\prime}\right)=\chi\left(\bar{U}_{j}\right)+1$.

DEFINITION. $\mathcal{L}^{\prime}$ as above is said to be a surgical modification of $\mathcal{L}$.

The principal results of this section will be the following propositions.

Proposition 1.3. If $\chi(M)=0$ and $\mathcal{L}=\left\{L_{1}, \ldots, L_{r}\right\}$ is an admissible system, then suitable surgical modifications on $\mathcal{L}$ produce an admissible system $\mathcal{L}^{\prime}=\left\{L_{1}^{\prime}, \ldots, L_{r}^{\prime}\right\}$ with the following properties:

(1) The tangent bundle to $\bigcup_{i=1}^{r} L_{i}^{\prime}$ extends to an $(n-1)$-plane field on $M$,

(2) $H^{1}\left(L_{i} ; \mathbf{R}\right) \neq 0,1<i<r$,

(3) if $n$ is odd, $\left\{\chi\left(L_{1}^{\prime}\right), \ldots, \chi\left(L_{r}^{\prime}\right)\right\}$ is a set of distinct negative integers.

Proposition 1.4. If $\mathcal{L}=\left\{L_{1}, \ldots, L_{r}\right\}$ is an admissible system, then $r<$ $\varphi(\alpha(M))-1$.

The second of these propositions is the heart of the proof of Theorem $A^{\prime}$, while the first, together with Thurston's results [6], makes possible a very straightforward construction of the foliated $n$-manifolds $\left(M_{k}^{n}, F_{k}\right)$ for Theorem B'.

First we will need a technical lemma. The notation $E \cdot N$ in the statement of the lemma denotes the usual dot product of $k$-tuples.

LeMmA 1.5. Let $\mathcal{E}^{k}=\left\{\left(\varepsilon_{1}, \ldots, \varepsilon_{k}\right): \varepsilon_{i} \in\{0,1,-1\}\right\}$. Given any $R>0$, any integer $k>1$, and any $\eta=\left(\eta_{1}, \ldots, \eta_{k}\right) \in\left(\mathbf{Z}_{2}\right)^{k}$, there exists $N=$ $\left(n_{1}, \ldots, n_{k}\right) \in \mathbf{Z}^{k}$ with all $n_{i}<0$ such that, for all $E, E^{\prime} \in \mathcal{E}^{k}$, 
(1) $E \cdot N=E^{\prime} \cdot N$ if and only if $E=E^{\prime}$,

(2) $|E \cdot N|>R$ if $E \neq(0, \ldots, 0)$,

(3) $N \equiv \eta \bmod 2$.

Proof. This is trivial for $k=1$, so we argue by induction on $k$. Suppose $N=\left(n_{1}, \ldots, n_{k}\right)$ satisfies the conditions. Choose $E^{*} \in \mathcal{E}^{k}$ such that $\left|E^{*} \cdot N\right|$ is maximal. Choose $n_{k+1}<0$ with the desired parity and such that $\left|n_{k+1}\right|>$ $2\left|E^{*} \cdot N\right|$. Let $N^{\prime}=\left(n_{1}, \ldots, n_{k+1}\right) \in \mathbf{Z}^{k+1}$. If $\sum_{i=1}^{k+1} \varepsilon_{i} n_{i}=\sum_{i=1}^{k+1} \varepsilon_{i}^{\prime} n_{i}, \varepsilon_{i}, \varepsilon_{i}^{\prime} \in$ $\{0,1,-1\}$, then

$$
\left(\varepsilon_{k+1}^{\prime}-\varepsilon_{k+1}\right) n_{k+1}=\sum_{i=1}^{k} \varepsilon_{i} n_{i}-\sum_{i=1}^{k} \varepsilon_{i}^{\prime} n_{i}=E \cdot N-E^{\prime} \cdot N .
$$

We consider the only possible cases:

(1) $\left|\varepsilon_{k+1}^{\prime}-\varepsilon_{k+1}\right|=1$,

(2) $\left|\varepsilon_{k+1}^{\prime}-\varepsilon_{k+1}\right|=2$,

(3) $\varepsilon_{k+1}^{\prime}=\varepsilon_{k+1}$ and $E \cdot N=E^{\prime} \cdot N$.

In. case (1), $\left|n_{k+1}\right|=\left|E \cdot N-E^{\prime} \cdot N\right|<2\left|E^{*} \cdot N\right|$ and this contradicts the assumption that $\left|n_{k+1}\right|>2\left|E^{*} \cdot N\right|$. In case (2), $\left|n_{k+1}\right|<\left|E^{*} \cdot N\right|$, again a contradiction. Thus case (3) alone is possible. By the inductive hypothesis, $E=E^{\prime}$ and so $\varepsilon_{i}=\varepsilon_{i}^{\prime}, 1<i<k+1$. Furthermore, if $E^{\prime \prime}=\left(\varepsilon_{1}^{\prime \prime}, \ldots, \varepsilon_{k+1}^{\prime \prime}\right)$ $\in \mathcal{E}^{k+1}$, we claim $\left|E^{\prime \prime} \cdot N^{\prime}\right|>R$. Indeed, if $\varepsilon_{k+1}^{\prime \prime}=0$, this comes from the inductive hypothesis and, if $\varepsilon_{k+1}^{\prime \prime} \neq 0$, then

$$
\begin{aligned}
\left|E^{\prime \prime} \cdot N^{\prime}\right| & =\left|\sum_{i=1}^{k} \varepsilon_{i}^{\prime \prime} n_{i} \pm n_{k+1}\right| \geqslant\left|n_{k+1}\right|-\left|\sum_{i=1}^{k} \varepsilon_{i}^{\prime \prime} n_{i}\right| \\
& >2\left|E^{*} \cdot N\right|-\left|E^{*} \cdot N\right|=\left|E^{*} \cdot N\right|>R .
\end{aligned}
$$

We also need the following fairly well-known fact.

LEMMA 1.6. If $W$ is a compact connected n-manifold, $v$ a vector field defined along $\partial W$ and transverse to $\partial W, \partial W_{+}$the part of $\partial W$ along which $v$ points out of $W, \partial W_{-}$the part along which $v$ points inward, then $v$ extends to a nowhere zero vector field on $W$ if and only if $\chi(W)=\chi\left(\partial W_{+}\right)=\chi\left(\partial W_{-}\right)$. Equivalently, for $n$ even, this holds precisely when $\chi(W)=0$, and for $n$ odd, it holds precisely when $\chi\left(\partial W_{+}\right)=\chi\left(\partial W_{-}\right)$.

Indeed, $v$ always extends to $\tilde{v}$ with only isolated nondegenerate singularities in $\operatorname{int}(W)$ and, $W$ being connected, the singularities can be eliminated if the sum of their indices $\iota(\tilde{v})=0$. The condition that $\iota(\tilde{v})=0$ is exactly the condition in (1.6), as is seen by standard relative Hopf index formulas (a very general formula is given by C. Pugh [4]).

Proof of (1.3). To begin with, note that adding a single handle to $L_{i}$ produces $L_{i}^{\prime}$ with $H^{1}\left(L_{1}^{\prime} ; \mathbf{R}\right) \neq 0$ and this property is never lost by adding 
more handles. This is easily established via the Mayer-Vietoris sequence. Thus, without loss of generality, we can suppose that $H^{1}\left(L_{i} ; \mathbf{R}\right) \neq 0,1 \leqslant i<$ $r$, and this property is never lost during the surgical modification, so property (2) is assured.

For the rest of the argument we must distinguish the cases in which $n$ is odd or even. First suppose that $n$ is even. Let $U_{1}, \ldots, U_{q}$ be the components of $M-\cup_{i=1}^{r} L_{i}$. Each $\bar{U}_{j}$ is a manifold with boundary $\partial \bar{U}_{j}$ equal to the union of some of the manifolds $L_{i}$. If $L_{i}, \ldots, L_{i p}$ are in $\operatorname{int}\left(\bar{U}_{j}\right)$, then $\bar{U}_{j}-\cup_{m=1}^{p} L_{i_{m}}$ is connected and we produce a compact connected manifold $W_{j}$ by cutting $\bar{U}_{j}$ open along each $L_{i_{m}}$. Note that $\chi\left(W_{j}\right)=\chi\left(\bar{U}_{j}\right)$. Also $0=\chi(M)=\sum_{j=1}^{q} \chi\left(\bar{U}_{j}\right)$ since the boundary components of every $\bar{U}_{j}$ are odd dimensional, hence have vanishing Euler characteristic.

Find a sequence $U_{n}, \ldots, U_{n_{p}}$ such that each $U_{i}$ appears at least once in the sequence and such that $\partial \bar{U}_{n_{j}}$ and $\partial \bar{U}_{n_{j+1}}$ have a common component $L_{n_{j}}$, $1<j<p-1$. (Repetitions have to be allowed. For instance, $\partial \bar{U}_{1}$ might contain every $L_{i}$, which would force such a sequence as $U_{1}, U_{2}, U_{1}, U_{3}$, $U_{1}, \ldots$ ) If $\chi\left(\vec{U}_{n_{1}}\right) \neq 0$, add a suitable number of handles to $L_{n_{1}}$ (into $\bar{U}_{n_{1}}$ if $\chi\left(\bar{U}_{n_{1}}\right)>0$, into $\bar{U}_{n_{2}}$ if $\left.\chi\left(\bar{U}_{n_{1}}\right)<0\right)$ so that the new $\chi\left(\bar{U}_{n_{1}}\right)=0$. Proceed in this way through the sequence. Since some $U_{n}$ may equal some $U_{n_{-1}}$, the good work already done on $U_{n_{j-1}}$ may be undone in passing from $U_{n_{j-1}}$ to $U_{n}$, but if $j<p$, this will be remedied in passing from $U_{n j}$ to $U_{n_{j+1}}$. When one finally arrives at $U_{n_{p}}$, every $U_{n g} \neq U_{n_{p}}$ satisfies $\chi\left(\bar{U}_{n g}\right)=0$. Assume $U_{n_{p}}=U_{q}$ and observe that

$$
0=\chi(M)=\sum_{j=1}^{q} \chi\left(\bar{U}_{j}\right)=\chi\left(\bar{U}_{q}\right)
$$

Thus, without loss of generality, we suppose $\mathcal{E}$ is such that each $\chi\left(W_{j}\right)=0$. Let $v$ be the unit normal field along $\cup_{i=1}^{r} L_{i}$. This defines a field $v_{j}$ along $\partial W_{j}$; hence (1.6) implies that $v$ extends to a nonsingular field on all of $M$. Property (1) follows and, since $n$ is even, property (3) is irrelevant.

Next suppose that $n$ is odd. Surgical modification reduces the Euler characteristic of $L_{i}$ by 2 for each handle added to $L_{i}$. In particular, this allows us to assume that $\chi\left(L_{i}\right)<0,1<i<r$. Remark also that for any compact odd-dimensional manifold $W, \chi(\partial W)$ is an even number since $\partial W$ is cobordant to zero. Let $\left\{\left[L_{1}\right], \ldots,\left[L_{k}\right]\right\}$ be a maximal linearly independent subset of [L]. If $\left[L_{i}\right]=\sum_{j=1}^{k} \varepsilon_{i}^{j}\left[L_{j}\right]$ then $L_{i}$, together with those $L_{j}$ for which $\varepsilon_{i}^{j} \neq 0$, cobound in $M$, so

$$
\chi\left(L_{i}\right) \equiv \sum_{j=1}^{k} \varepsilon_{i}^{j} \chi\left(L_{j}\right) \bmod 2
$$


Let $R>\left|\chi\left(L_{i}\right)\right|, 1 \leqslant i \leqslant r$, and let $N=\left(n_{1}, \ldots, n_{k}\right)$ satisfy (1.5) with $n_{j} \equiv$ $\chi\left(L_{j}\right) \bmod 2,1 \leqslant j<k$. Let $\sigma_{1}, \ldots, \sigma_{k}$ be circles in $M$ such that $\left[\sigma_{i}\right] *\left[L_{j}\right]=$ $\delta_{i j}, 1<i, j<k$. Let $\gamma=\sum_{j=1}^{k} n_{j}\left[\sigma_{j}\right]$. Then, if $E_{i}=\left(\varepsilon_{i}^{1}, \ldots, \varepsilon_{i}^{k}\right)$ denotes the coefficient $k$-tuple of $\left[L_{i}\right]$, we see that $\gamma *\left[L_{i}\right]=E_{i} \cdot N$. Since $E_{i} \neq \pm E_{j}$ for $i \neq j$, (1.5) implies that $\left|\gamma *\left[L_{i}\right]\right|, 1 \leqslant i \leqslant k$, are distinct nonzero integers. Changing the orientation of $L_{i}$, if necessary, we can assume all $\gamma *\left[L_{i}\right]$ are negative. By the above,

$$
\left|\gamma *\left[L_{i}\right]\right|>\left|\chi\left(L_{i}\right)\right|, \quad \gamma *\left[L_{i}\right] \equiv \chi\left(L_{i}\right) \bmod 2, \quad 1 \leqslant i \leqslant r .
$$

Thus, by surgical modification, we can produce $\mathcal{L}^{\prime}$ such that $\gamma *\left[L_{i}\right]=$ $\gamma *\left[L_{i}^{\prime}\right]=\chi\left(L_{i}^{\prime}\right)<0,1 \leqslant i<r$, and these integers are distinct.

Let $\xi \in H^{n-1}(M)$ be the Poincare dual of $\gamma$. Then, $\xi\left(\left[L_{i}\right]\right)=\int_{L_{1}} \xi=\chi\left(L_{i}\right)$. Let $v$ be the unit normal field along $\cup_{i=1}^{r} L_{i}^{\prime}$ directed in the positive sense (relative to the orientation of $L_{i}^{\prime}$ ). Let $U_{1}, \ldots, U_{q}$ be the components of $M-\cup_{i=1}^{r} L_{i}^{\prime}$ and let $\xi_{i}$ be the restriction of $\xi$ to $\vec{U}_{i}$. On $\partial \bar{U}_{i}$, understood as oriented inward, $\xi_{i}$ integrates to 0 ; hence on $\partial \vec{U}_{i+}$, oriented outward, $\xi_{i}$ integrates to the same value as on $\partial \bar{U}_{i-}$, oriented inward. That is,

$$
\chi\left(\partial U_{i+}\right)=\chi\left(\partial U_{i-}\right), \quad 1 \leqslant i \leqslant q .
$$

Let $W_{i}$ be the compact connected manifold obtained by cutting $\bar{U}_{i}$ open along each $L_{j}^{\prime} \subset \operatorname{int}\left(\bar{U}_{i}\right)$. Then $v$ defines $v_{i}$ along $\partial W_{i}$ and transverse to $\partial W_{i}$. Each $L_{j}^{\prime} \subset \operatorname{int}\left(\bar{U}_{i}\right)$ determines two diffeomorphic boundary components of $W_{i}$, one in $\partial W_{i+}$ and one in $\partial W_{i-}$. Thus $\chi\left(\partial W_{i+}\right)=\chi\left(\partial W_{i-}\right), 1 \leqslant i \leqslant q$, so $v_{i}$ extends to a nonsingular vector field over $W_{i}$ (by (1.6)); hence $v$ extends to a nonsingular vector field over $M$. This defines the desired extension of the tangent bundle of $\cup_{i=1}^{r} L_{i}^{\prime}$, hence establishes property (1). Properties (2) and (3) have already been assured, so the proof of (1.3) is complete.

The proof of (1.4) will proceed by a sequence of lemmas. We will be interested only in the separation properties of $\mathcal{L}$ in $M$, so all homological arguments will be carried out with $\mathbf{Z}_{2}$-coefficients. This is legitimate by reason of (1.2) and the remark following that lemma. Thus, $\operatorname{rank}(\mathcal{L})$ is $\operatorname{dim}\left(\operatorname{span}[\mathcal{E}]_{2}\right)$ and the coefficient $k$-tuple $E_{j}$ for $\left[L_{j}\right]_{2}$ will be an element of $\left(Z_{2}\right)^{k}$.

Definition. If $\mathcal{L}$ and $\mathcal{L}^{\prime}$ are admissible systems, then $\mathcal{L}<\mathcal{L}^{\prime}$ means $\mathcal{L} \subset \mathcal{L}^{\prime}$ and $\operatorname{rank}(\mathcal{L})=\operatorname{rank}\left(\mathcal{L}^{\prime}\right)$.

Definition. An admissible system $\mathcal{E}=\left\{L_{1}, \ldots, L_{r}\right\}$ is polygonal if each $L_{i}$ occurs as a boundary component of some $U_{j}$ (hence of exactly two) where $U_{1}, \ldots, U_{q}$ is the set of components of $M-\bigcup_{i=1}^{r} L_{i}$.

The term "polygonal" is due to a heuristic procedure by which each $\bar{U}_{j}$ is represented by a diagram such as 


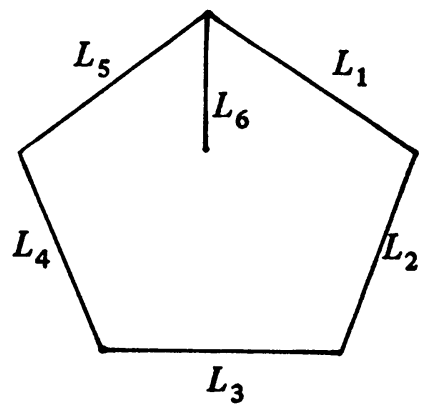

where $\partial \bar{U}_{j}=L_{1} \cup L_{2} \cup L_{3} \cup L_{4} \cup L_{5}$ and $L_{6} \subset \operatorname{int}\left(\bar{U}_{j}\right)$. The word "polygonal" indicates that each $\bar{U}_{j}$ is represented by a simple closed polygon, hence has no $L_{i}$ in the interior.

In the above example, produce $L_{1}^{\prime}$ and $L_{6}^{\prime} \subset \operatorname{int}\left(U_{j}^{\prime}\right)$ by displacing $L_{1}$ and $L_{6}$ a small distance $\varepsilon>0$ along normal trajectories. A schematic representation of this situation would be

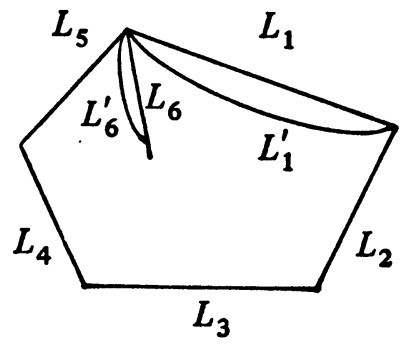

since $L_{i}$ and $L_{i}^{\prime}$ cobound for $i=1,6$. A connected sum of $L_{1}^{\prime}$ and $L_{6}^{\prime}$ along a small tube lying entirely in $\operatorname{int}\left(\bar{U}_{j}\right)$ and missing $L_{6}$ produces $L \subset \operatorname{int}\left(\bar{U}_{j}\right)$ such that $L \cap L_{6}=\varnothing$ and $L \cup L_{6}$ disconnects $\bar{U}_{j}$. The schematic representation of the final state of affairs would be

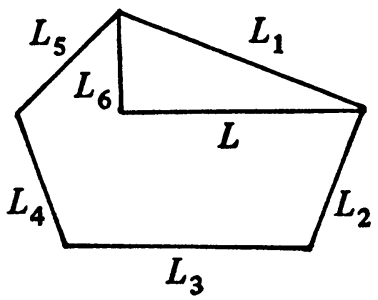

Finally, if $\left[L_{2} \neq\left[L_{i}\right]_{2}\right.$ for every $L_{i} \in \mathcal{L}$, this construction produces $\mathcal{L}^{\prime}=$ $\left\{L_{1}, \ldots, L_{r}, L\right\}$ with $\mathcal{L}<\mathscr{L}^{\prime}$. This idea is exploited in the following lemma.

LEMMA 1.7. If $\mathcal{L}$ is an admissible system of rank at least 2 , then $\mathcal{L}<\mathfrak{L}^{\prime}$ for some admissible polygonal system $\mathfrak{L}^{\prime}$.

Proof. As usual, arrange that $\left\{\left[L_{1}\right]_{2}, \ldots,\left[L_{k}\right]_{2}\right\}$ is a maximal linearly independent subset of $[\mathcal{L}]_{2}$. If $L_{i} \subset \operatorname{int}\left(\bar{U}_{j}\right)$, then $L_{1} \cup \cdots \cup L_{k} \cup L_{i}$ cannot 
separate $M$, hence $i<k$. Arrange that $\left\{L_{1}, \ldots, L_{p}\right\}, p<k$, is the subset of those $L_{i}$ each of which is contained in the interior of some $\bar{U}_{j}$. Thus every $E_{j}=\left(\varepsilon_{j}^{1}, \ldots, \varepsilon_{j}^{k}\right)$ has $\varepsilon_{j}^{i}=0$ for $1 \leqslant i<p$. The system $\mathcal{E}$ is polygonal if and only if $p=0$.

If $p=k$, then $k=r \geqslant 2$ and by (1.1) the class $x=\left[L_{1}\right]_{2}+\cdots+\left[L_{r}\right]_{2}$ can be represented by connected orientable $L_{r+1} \subset M$ disjoint from every $L_{i}$, $1<i<r,\left[L_{r+1}\right]_{2}$ being linearly independent of $\left[L_{i}\right]_{2}, 1 \leqslant i \leqslant r . \mathcal{L}^{\prime}=$ $\left\{L_{1}, \ldots, L_{r}, L_{r+1}\right\}$ is admissible of rank $k=r$ and is also polygonal.

If $0<p<k$, let $U_{i}$ be the component such that $L_{p} \subset \operatorname{int}\left(\bar{U}_{i}\right)$. Let $L_{s} \subset \partial \bar{U}_{i}$. As in the remarks preceding the lemma, displace both $L_{s}$ and $L_{p}$ slightly and form a connected sum so as to produce $L_{r+1} \subset \operatorname{int}\left(\bar{U}_{i}\right)$ with

$$
\begin{gathered}
L_{r+1} \cap L_{j}=\varnothing, \quad 1<j<r, \\
{\left[L_{r+1}\right]_{2}=\left[L_{p}\right]_{2}+\left[L_{s}\right]_{2} .}
\end{gathered}
$$

This gives an admissible system $\left\{L_{1}, \ldots, L_{r+1}\right\}$ of rank $k$ in which $\left\{L_{1}, \ldots, L_{p-1}\right\}$ is the subset of those $L_{i}$ contained in some $\operatorname{int}\left(\bar{U}_{j}\right)$. This process, repeated finitely often, produces the desired polygonal system $\mathcal{L}^{\prime}$.

Remark that polygonal systems must have rank at least 2 , hence the necessity of the restriction on $\operatorname{rank}(\mathcal{E})$ in (1.7). This in turn is the reason why $\varphi(k)$ must be defined by a different formula when $k=0,1$.

Definition. An admissible system is triangular if it is polygonal and if each $\partial \bar{U}_{j}$ has exactly three components.

LEMMA 1.8. Let $\mathcal{E}$ be an admissible polygonal system. Then $\mathcal{L}<\mathcal{L}^{\prime}$ for some admissible triangular system.

Proof. If some $\partial\left(\bar{U}_{j}\right)$ has more than three components (fewer are impossible by the definition of an admissible system), let $L_{1}, L_{2}, L_{3}, L_{4}$ be distinct components of $\partial \bar{U}_{j}$. If both $\left\{\left[L_{1}\right]_{2},\left[L_{2}\right]_{2},\left[L_{3}\right]_{2}\right\}$ and $\left\{\left[L_{2}\right]_{2},\left[L_{3}\right]_{2},\left[L_{4}\right]_{2}\right\}$ are linearly dependent sets, then $\left[L_{1}\right]_{2}=\left[L_{2}\right]_{2}+\left[L_{3}\right]_{2}$ and $\left[L_{4}\right]_{2}=\left[L_{2}\right]_{2}+\left[L_{3}\right]_{2}$, and this would contradict $\left[L_{1}\right]_{2} \neq\left[L_{4}\right]_{2}$. Thus we can assume that $\left\{\left[L_{1}\right]_{2}\right.$, $\left.\left[L_{2}\right]_{2},\left[L_{3}\right]_{2}\right\}$ is a linearly independent set. We claim that either $\left[L_{1}\right]_{2}+\left[L_{2}\right]_{2}$ or $\left[L_{1}\right]_{2}+\left[L_{3}\right]_{2}$ is not in $[\mathcal{L}]_{2}$. Indeed, if one can find $L_{p}, L_{q} \in \mathcal{L}$ such that $\left[L_{p}\right]_{2}=\left[L_{1}\right]_{2}+\left[L_{2}\right]_{2},\left[L_{q}\right]_{2}=\left[L_{1}\right]_{2}+\left[L_{3}\right]_{2}$, then $p, q \neq 1,2,3$ and $p \neq q$. Thus $\left\{L_{1}, L_{2}, L_{3}, L_{p}, L_{q}\right\}$ is an admissible system of rank 3 . The complement in $M$ of $L_{1} \cup L_{2} \cup L_{p}$ has two components. Neither $L_{3}$ nor $L_{q}$ individually disconnects the component in which it lies, but $L_{1} \cup L_{3} \cup L_{q}$ disconnects $M$, so $L_{3}$ and $L_{q}$ must lie in the same component of $M-L_{1}-L_{2}-L_{p}$ and together disconnect it into two components, one of which must have boundary $L_{1} \cup L_{3} \cup L_{q}$ (because all other possibilities lead to a linear dependence between $\left[L_{1}\right]_{2}$ and $\left.\left[L_{2}\right]_{2}\right)$. Thus $M-\left(L_{1} \cup L_{2} \cup L_{3} \cup L_{p} \cup L_{q}\right)$ 
has components $W_{1}, W_{2}, W_{3}$ with

$$
\begin{aligned}
& \partial \bar{W}_{1}=L_{1} \cup L_{3} \cup L_{q}, \\
& \partial \bar{W}_{2}=L_{3} \cup L_{q} \cup L_{2} \cup L_{p}, \\
& \partial \bar{W}_{3}=L_{1} \cup L_{2} \cup L_{p} .
\end{aligned}
$$

Since $\mathcal{L}$ contains this admissible system, $U_{j}$ must be contained in one of $W_{1}$, $W_{2}, W_{3}$. But $\bar{U}_{j} \subset \bar{W}_{1}$ contradicts $L_{2} \not \subset \bar{W}_{1}, \bar{U}_{j} \subset \bar{W}_{2}$ contradicts $L_{1} \not \bar{W}_{2}$, and $\bar{U}_{j} \subset \bar{W}_{3}$ contradicts $L_{3} \subset \bar{W}_{3}$. Thus we can assume that no $L_{p} \in \mathcal{L}$ has $\left[L_{p}\right]_{2}=\left[L_{1}\right]_{2}+\left[L_{2}\right]_{2}$. Thus, displace both $L_{1}$ and $L_{2}$ to the interior of $U_{j}$ and form their connected sum, producing $L_{r+1} \subset U_{j}$ which separates $\bar{U}_{j}$ into two components, one with boundary $L_{r+1} \cup L_{1} \cup L_{2}$, the other with one fewer boundary component than $\bar{U}_{j}$ had. By the above, $\left\{L_{1}, \ldots, L_{r}, L_{r+1}\right\}$ is admissible, polygonal, and has the same rank as $\mathcal{L}$. Finite iteration of this process will finally yield the desired triangular system.

Thus every admissible $\mathcal{L}$ can be extended to an admissible triangular system $L^{\prime}$ of the same rank. The next project is to produce a formula for the number of elements in $\mathcal{L}^{\prime}$ in terms of the integer $\operatorname{rank}\left(\mathcal{L}^{\prime}\right)$.

LEMMA 1.9. Let $W$ be a compact, orientable, connected manifold and suppose that $\partial W$ has $h$ components, $h>3$. Let $L_{1}, \ldots, L_{p} \subset \operatorname{int}(W)$ be disjoint, closed, connected, orientable submanifolds of $\operatorname{int}(W)$ of codimension one. Suppose that $\left\{L_{1}, \ldots, L_{s}\right\}$ is a maximal subset such that $W-\cup_{i=1}^{s} L_{i}$ is connected. Suppose that, for each component $U$ of $W-\cup_{i=1}^{p} L_{i}$, the manifold $\partial \bar{U}$ has three components and that every $L_{i}$ occurs as some boundary component for some $U$. Then $p=h+3 s-3$.

Proof. We do a double induction on $h>3$ and $s>0$. If $h=3$ and $s=0$, we claim that $p=0$. Otherwise, $L_{1}$ separates $W$ into two components one of which, say $W^{\prime}$, must be bounded by $L_{1}$ and a single component of $\partial W$. If there is any $L_{i} \subset W^{\prime}$, then, since $s=0$, we again get a component of $W-\left(L_{1} \cup L_{i}\right)$ with two boundary components. Proceeding in this way, we finally see that some component of $W-\cup_{i=1}^{p} L_{i}$ has just two boundary components, contrary to our hypothesis.

Suppose the assertion is true for $s=0$ and for all $h$ such that $3<h<m$. If $\partial W$ has $m$ components and $s=0$, then $L_{1}$ divides $W$ into two components each of which has at least three boundary components (for the same reason as above). If the number of boundary components in each is $h_{1}$ and $h_{2}$ respectively, then $3<h_{i}<m, i=1,2$, and $h_{1}+h_{2}=m+2$. But each of $L_{2}, \ldots, L_{p}$ belongs to one or another component of $W-L_{1}$ and these components are divided into triangular components. The inductive hypothesis implies that 


$$
p-1=h_{1}-3+h_{2}-3=h_{1}+h_{2}-6=m-4
$$

and so $p=m-3$ as desired.

Finally, assume the assertion for $0 \leqslant s<n$ and for all $h$. Suppose $\left\{L_{1}, \ldots, L_{n}\right\}$ is a maximal subset not separating $W$. Necessarily $n<p$ since, otherwise, $h=3, p=0$, and so $n=0$. Thus, the set $\left\{L_{1}, \ldots, L_{n}, L_{n+1}\right\}$ separates $W$ into two components $W_{1}$ and $W_{2}$. We can suppose that the common boundary is $\partial W_{1} \cap \partial W_{2}=L_{1} \cup \cdots \cup L_{q} \cup L_{n+1}$ where $0 \leqslant q \leqslant$ $n$. We can even suppose $q>0$ since, if this were impossible to arrange, none of $L_{1}, \ldots, L_{n}$ could ever occur as a boundary component of any component of $W-\cup_{i=1}^{p} L_{i}$, contradicting the assumption that $n>0$. Suppose that $s_{1}$ of $\left\{L_{q+1}, \ldots, L_{n}\right\}$ fall into $W_{1}$ and that $s_{2}$ of them fall into $W_{2}$. Evidently these $s_{i}$ manifolds are maximal nonseparating in $W_{i}$ and $s_{i}<n, i=1,2$. Let $h_{i}$ be the number of boundary components of $W_{i}$ and $p_{i}$ the number of $L_{j} \subset$ $\operatorname{int}\left(W_{i}\right), i=1,2$. By the inductive hypothesis $p_{i}=h_{i}+3 s_{i}-3, i=1,2$. But $h_{1}+h_{2}=h+2 q+2, s_{1}+s_{2}+q=n, p_{1}+p_{2}=p-q-1$, and so

$$
\begin{aligned}
p & =p_{1}+p_{2}+q+1=\left(h_{1}+3 s_{1}-3\right)+\left(h_{2}+3 s_{2}-3\right)+q+1 \\
& =(h+2 q+2)+3 s_{1}+3 s_{2}+q-5 \\
& =h+3\left(s_{1}+s_{2}+q\right)-3=h+3 n-3 .
\end{aligned}
$$

LEMMA 1.10. Let $\mathcal{L}=\left\{L_{1}, \ldots, L_{r}\right\}$ be an admissible triangular system of rank $k$. Then $r=3 k-3$.

Proof. Let $U$ be any component of $M-\cup_{i=1}^{r} L_{i}$ and let $\partial \bar{U}=L_{1} \cup L_{2} \cup$ $L_{r}$. Let $W_{1}=\bar{U}$ and $W_{2}=M-U$. These are compact manifolds each with three boundary components. Also, $W_{2}$ is connected since $\left\{\left[L_{1}\right]_{2},\left[L_{2}\right]_{2},\left[L_{r}\right]_{2}\right\}$ is pairwise linearly independent. The set $\left\{\left[L_{1}\right]_{2},\left[L_{2}\right]_{2}\right\}$ extends to a maximal independent set $\left\{\left[L_{1}\right]_{2}, \ldots,\left[L_{k}\right]_{2}\right\}$. If $i \neq 1,2, r$, then $L_{i} \subset \operatorname{int}\left(W_{2}\right)$ and $\left\{L_{3}, \ldots, L_{k}\right\}$ is a maximal nonseparating subset in $W_{2}$. Thus, by (1.9), $r-3=3+3(k-2)-3=3 k-6$ and so $r=3 k-3$.

We now prove (1.4). If $\mathcal{E}=\left\{L_{1}, \ldots, L_{r}\right\}$ is an admissible system of rank $k$, let $\left\{\left[L_{1}\right], \ldots,\left[L_{k}\right]\right\}$ be a maximal independent subset. We emphasize the return to integral homology. By (1.1), this set spans a direct summand of $H_{n-1}(M)$. If $x_{i}$ denotes the Poincaré dual of $\left[L_{i}\right], 1 \leqslant i \leqslant k$, then $\left\{x_{1}, \ldots, x_{k}\right\}$ extends to a basis of $H^{1}(M)$. But $x_{i} \cup x_{j}$ is the Poincare dual of $\left[L_{i}\right] *\left[L_{j}\right]=0$; hence $k \leqslant \alpha(M)$. If $k=0$, then $r=0$ and there is nothing to prove. If $k=1$, then $r=1$ and $1 \leqslant \alpha(M)$, so $r=\varphi(1)-1 \leqslant \varphi(\alpha(M))-1$. If $k>2$, then (1.7), (1.8) and (1.10) together imply

$$
r<3 k-3=\varphi(k)-1 \leqslant \varphi(\alpha(M))-1 \text {. }
$$

2. Applications to foliated $n$-manifolds. It is easy to prove Theorem $A^{\prime}$ as a corollary to (1.4). Let $(M, F)$ be a foliated $n$-manifold with $h(F)=r$. Let 
$\left\{L_{1}, \ldots, L_{r}\right\}$ be a set of closed leaves of $F$ such that $\left\{\left[L_{1}\right], \ldots,\left[L_{r}\right]\right\}$ are distinct modulo sign. If no $\left[L_{i}\right]=0$, then $\left\{L_{1}, \ldots, L_{r}\right\}$ is an admissible system. If, say, $\left[L_{r}\right]=0$, then $\left\{L_{1}, \ldots, L_{r-1}\right\}$ is admissible. In the first case, $r<\varphi(\alpha(M))-1$, and in the second, $r-1 \leqslant \varphi(\alpha(M))-1$. In any case, $h(F)<\varphi(\alpha(M))$. Also, by (1.2), $h_{2}(F)=h(F)$.

If $L$ and $L^{\prime}$ are closed leaves of $F$ with $|\chi(L)| \neq\left|\chi\left(L^{\prime}\right)\right|$, then $[L] \neq \pm\left[L^{\prime}\right]$. Indeed, let $c \in H^{n-1}(M)$ be the Euler class of the tangent bundle to $F$. Then, if $[L]= \pm\left[L^{\prime}\right]$, we would have

$$
\pm \chi(L)=c[L]= \pm c\left[L^{\prime}\right]= \pm \chi\left(L^{\prime}\right)
$$

a contradiction. It follows that $e(F) \leqslant h(F)$ and the proof of Theorem $\mathrm{A}^{\prime}$ is complete. As remarked in the introduction, Theorem $\mathrm{A}$ is a trivial consequence when $\operatorname{dim}(M)=3$.

Definition. An admissible system $\mathcal{L}$ is integrable if there is a foliation $F$ of $M$ with each $L_{i} \in \mathcal{L}$ as a leaf. (Note that the orientation of $L_{i}$ as a leaf of $F$ may not be the same as that of $L_{i} \in \mathcal{L}$.)

THEOREM 2.1. If $\chi(M)=0$ and $\mathfrak{L}$ is admissible, then a suitable surgical modification on $\mathcal{L}$ produces an integrable $\mathcal{L}^{\prime}$. If, in addition, $\operatorname{dim}(M)$ is odd, it can be arranged that the Euler characteristics of the elements of $\mathcal{L}^{\prime}$ form a set of distinct negative integers.

Proof. We allow $\mathcal{L}=\varnothing$, in which case $\operatorname{rank}(\mathcal{L})=0$. Produce $\mathscr{L}^{\prime}$ satisfying (1.3). Then, by Thurston ([6], relative version if $\operatorname{rank}(\mathcal{L}) \neq 0$ ), there is a foliation of $M$ having each $L_{i}^{\prime} \in \mathfrak{L}^{\prime}$ as a leaf.

Corollary 2.2. If $\chi(M)=0$ and $M$ supports an admissible system of rank $k$, then $M$ admits a foliation $F$ with $h(F)>\varphi(k)$. (Here we allow an empty admissible system; hence $k=0$ ). If $\operatorname{dim}(M)$ is odd, such $F$ can also be chosen so that $e(F)>\varphi(k)$.

Proof. If $k>2$, then by (1.7), (1.8), and (1.10) there is an admissible system $\mathcal{L}$ of cardinality $3 k-3$. In any case, there is such a system $\mathcal{L}$ of cardinality $\varphi(k)-1$. By $(2.1)$ we can assume the existence of a foliation $F$ having each $L \in \mathcal{L}$ as a leaf. Also, on odd-dimensional manifolds it can be arranged that the Euler characteristics $\chi(L), L \in \mathcal{L}$, form a set of distinct negative integers. By the Reeb stability theorem we can assume that some leaf is noncompact; hence standard methods show that there is a closed transversal to $F$ missing every leaf $L \in \mathcal{L}$. Modifying $F$ along this transversal [7] produces a foliation $F^{\prime}$ in which each element of $\mathcal{L}$ is a leaf, but which also has a homologically trivial closed leaf with Euler characteristic zero. Thus $h\left(F^{\prime}\right)>\varphi(k)$, and on odd dimensional manifolds $M$, one also has $e\left(F^{\prime}\right)>$ $\varphi(k)$.

In order to prove Theorem $\mathrm{B}^{\prime}$, we will now construct a suitable $M_{k}^{n}$ and 
apply the above corollary together with Theorem $\mathrm{A}^{\prime}$.

First suppose that $n$ is odd and at least equal to 3. Let $M_{0}^{n}=S^{n}$ and $M_{1}^{n}=T^{n}$. Evidently $\alpha\left(S^{n}\right)=0$ and $\alpha\left(T^{n}\right)=1$. For $k>2$, let $W_{k}^{n}$ be the complement in $D^{n}$ of $k$ disjoint open balls with closures in the interior of $D^{n}$ and let $M_{k}^{n}$ be the double of $W_{k}^{n}$. By the above corollary and the obvious fact that $M_{k}^{n}$ supports an admissible system $\left\{L_{1}, \ldots, L_{k}\right\}$ of rank $k$, we see that $M_{k}^{n}$ supports a foliation $F_{k}$ with $h\left(F_{k}\right)>e\left(F_{k}\right) \geqslant \varphi(k)$.

If $n$ is even and at least equal to 4 , and if $k>0$, set $M_{k}^{n}=M_{k}^{n-1} \times S^{1}$. If $\left\{L_{1}, \ldots, L_{k}\right\}$ is an admissible system of rank $k$ on $M_{k}^{n-1}$, then the manifolds $L_{i}^{\prime}=L_{i} \times S^{1} \subset M_{k}^{n}, 1<i<k$, form an admissible system of rank $k$. For the case $k=0$, set $M_{0}^{6}=S^{3} \times S^{3}$ and $M_{0}^{n}=S^{3} \times S^{3} \times S^{n-6}, n>6$. In all cases, $\chi\left(M_{k}^{n}\right)=0$ and the above corollary guarantees the existence of a foliation $F_{k}$ with $h\left(F_{k}\right)>\varphi(k)$.

The following lemma together with Theorem $\mathrm{A}^{\prime}$ clearly completes the proof of Theorem $\mathrm{B}^{\prime}$. As remarked in the introduction, when $n=3$ we get Theorem $B$ as a special case.

LEMMA 2.3. $\alpha\left(M_{k}^{n}\right)=k$ for all $n>3$ and all $k>0$ (excepting, of course, the nonexistent $\left.M_{0}^{4}\right)$.

Proof. For $k=0,1$ this is obvious. For $n$ odd and $k \geqslant 2, \pi_{i}\left(W_{k}^{n}\right)=0$, $0<i<n-2$, so the Hurewicz theorem gives

$$
H_{n-1}\left(W_{k}^{n}\right)=\pi_{n-1}\left(W_{k}^{n}\right)=\mathbf{Z}^{k} .
$$

There is an exact Mayer-Vietoris sequence

$$
\begin{gathered}
H_{n}\left(W_{k}^{n}\right) \oplus H_{n}\left(W_{k}^{n}\right) \rightarrow H_{n}\left(M_{k}^{n}\right) \rightarrow H_{n-1}\left(\partial W_{k}^{n}\right) \rightarrow H_{n-1}\left(W_{k}^{n}\right) \\
\oplus H_{n-1}\left(W_{k}^{n}\right) \rightarrow H_{n-1}\left(M_{k}^{n}\right) \rightarrow H_{n-2}\left(\partial W_{k}^{n}\right) .
\end{gathered}
$$

But $H_{n}\left(W_{k}^{n}\right)=0, H_{n}\left(M_{k}^{n}\right)=\mathbf{Z}, H_{n-1}\left(\partial W_{k}^{n}\right)=\mathbf{Z}^{k+1}, H_{n-1}\left(W_{k}^{n}\right)=\mathbf{Z}^{k}$ and $H_{n-2}\left(\partial W_{k}^{n}\right)=0$, so we get

$$
0 \rightarrow \mathbf{Z} \rightarrow \mathbf{Z}^{k+1} \rightarrow \mathbf{Z}^{2 k} \rightarrow H_{n-1}\left(M_{k}^{n}\right) \rightarrow 0 ;
$$

hence $0 \rightarrow \mathbf{Z}^{k} \rightarrow \mathbf{Z}^{2 k} \rightarrow H_{n-1}\left(M_{k}^{n}\right) \rightarrow 0$. As earlier remarked, $H_{n-1}\left(M_{k}^{n}\right)$ is free abelian, so the sequence splits and $\mathbf{Z}^{2 k} \cong \mathbf{Z}^{k} \oplus H_{n-1}\left(M_{k}^{n}\right)$. Therefore $H_{n-1}\left(M_{k}^{n}\right) \cong \mathbf{Z}^{k}$ for $n$ odd. The admissible system $\left\{L_{1}, \ldots, L_{k}\right\}$ provides a free basis of $H_{n-1}\left(M_{k}^{n}\right)$ with all intersection products zero. By Poincaré duality, we get a basis $x_{1}, \ldots, x_{k}$ of $H^{1}\left(M_{k}^{n}\right)$ with all $x_{i} \cup x_{j}=0$, so $\alpha\left(M_{k}^{n}\right)=k$.

If $n$ is even, then

$$
H_{n-1}\left(M_{k}^{n}\right) \approx H_{n-1}\left(M_{k}^{n-1}\right) \oplus H_{n-1}\left(M_{k}^{n-1}\right) \cong \mathrm{Z}^{k+1} .
$$

Again we get classes $x_{1}, \ldots, x_{k} \in H^{1}\left(M_{k}^{n}\right)$ with all $x_{i} \cup x_{j}=0$. These extend to a basis by adjunction of the class $x_{k+1}$ defined by the projection map 
of $M_{k}^{n}$ onto the factor $S^{\prime}$, and $x_{i} \cup x_{k+1} \neq 0,1 \leqslant i \leqslant k$. It is again clear that $\alpha\left(M_{k}^{n}\right)=k$.

3. A class of examples. Let $T_{g}$ denote the closed oriented surface of genus $g$. Let $E_{g, n}$ denote the total space of the oriented $S^{1}$-bundle over $T_{g}$ having Euler class $n \in H^{2}\left(T_{g}\right)=\mathbf{Z}$. Since $E_{g, n} \cong E_{g,-n}$, we will always take $n>0$. Remark, in particular, that $E_{0,0}=S^{1} \times S^{2}, E_{0,1}=S^{3}$ and $E_{1,0}=T^{3}$.

We will compute the following.

$$
\alpha\left(E_{g, n}\right)= \begin{cases}1, & n=g=0 \\ 2 g, & n=1, \\ g, & \text { otherwise }\end{cases}
$$

Indeed, $H^{1}\left(E_{0,0}\right)=\mathbf{Z}$, so the first equality is immediate.

Since the bundle $S^{1} \hookrightarrow E_{g, n} \rightarrow_{\pi} T_{g}$ is orientable, the cohomology spectral sequence has ordinary coefficients. Since $H^{1}\left(T_{g}\right)=\mathbf{Z}^{2 g}$, the $E_{2}$ stage takes the following form.

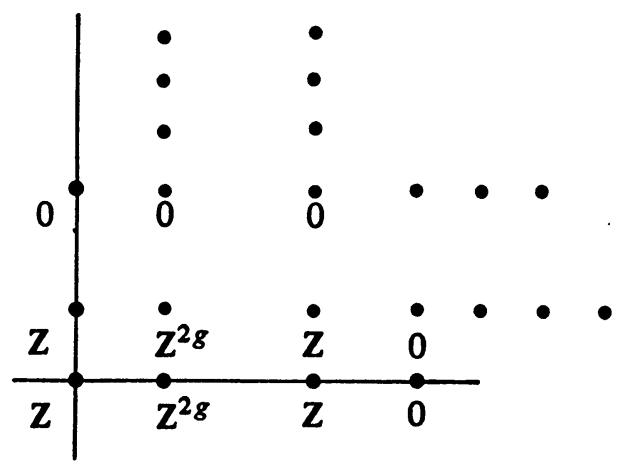

If $1 \in \mathrm{Z}=E_{2}^{0,1}=H^{1}\left(S^{1}\right)$ corresponds to the orientation of the fiber, then

$$
d_{2}(1)=n \in \mathrm{Z}=E_{2}^{2,0}=H^{2}\left(T_{g}\right)
$$

is the Euler class of the bundle. It is also clear that $E_{3}=E_{\infty}$.

LEMMA 3.2. $H^{1}\left(E_{g, 1}\right)=\mathbf{Z}^{2 g}$ and the cup products of elements in this group are all zero. Thus $\alpha\left(E_{g, 1}\right)=2 g$.

Proof. In the spectral sequence, $d_{2}(1)=1$ and so $d_{2}: E_{2}^{0,1} \rightarrow E_{2}^{2,0}$ is an isomorphism. Thus, $E_{3}^{0,1}=0$ and $E_{3}^{1,0}=\mathbf{Z}^{28}$. Since $E_{3}=E_{\infty}$, we have $H^{1}\left(E_{g, 1}\right)=\mathrm{Z}^{28}$. Indeed, by the edge homomorphism, $\pi^{*}: H^{1}\left(T_{g}\right) \rightarrow H^{1}\left(E_{g, 1}\right)$ is an isomorphism. Since $E_{3}^{2,0}=0$, the edge homomorphism also shows that $\pi^{*}: H^{2}\left(T_{g}\right) \rightarrow H^{2}\left(E_{g, 1}\right)$ is trivial. It follows that all cup products of elements in $H^{1}\left(E_{g, 1}\right)$ are trivial.

LEMMA 3.3. If $n>1$, then $H^{1}\left(E_{g, n}\right)=\mathbf{Z}^{2 g}, H^{2}\left(E_{g, n}\right)=\mathbf{Z}^{28} \oplus \mathbf{Z}_{n}$, and there is a basis $x_{1}, \ldots, x_{2 g}$ of $H^{1}\left(E_{g, n}\right)$ such that 
(1) $x_{i} \cup x_{g+i}=x_{j} \cup x_{g+j}$ is a generator of $\mathbf{Z}_{n} \subset H^{2}\left(E_{g, n}\right), 1<i, j<g$,

(2) $x_{i} \cup x_{j}=0$ if $|i-j| \neq g$.

Proof. In the spectral sequence, $d_{2}(1)=n$, so $E_{3}^{0,1}=0, E_{3}^{2,0}=\mathrm{Z}_{n}, E_{3}^{1,0}=$ $E_{3}^{1,1}=\mathrm{Z}^{28}$. By the edge homomorphism, $\pi^{*}: H^{1}\left(T_{g}\right) \rightarrow H^{1}\left(E_{g, n}\right)$ is an isomorphism, the group being $\mathbf{Z}^{28}$, and $\pi^{*}: H^{2}\left(T_{8}\right) \rightarrow H^{2}\left(E_{g, n}\right)$ has image $\mathbf{Z}_{n}$. Also, there is an exact sequence

$$
0 \rightarrow \mathbf{Z}_{n} \rightarrow H^{2}\left(E_{g, n}\right) \rightarrow \mathbf{Z}^{28} \rightarrow 0
$$

so $H^{2}\left(E_{g, n}\right)=\mathbf{Z}_{n} \oplus \mathbf{Z}^{28}$. Finally, $H^{1}\left(T_{g}\right)$ has a basis $y_{1}, \ldots, y_{2 g}$ such that, for a generator $z \in H^{2}\left(T_{g}\right)$,

$$
\begin{aligned}
y_{i} \cup y_{g+i} & =z, & 1<i<g \\
y_{i} \cup y_{j} & =0, & |i-j| \neq g .
\end{aligned}
$$

Setting $x_{i}=\pi^{*}\left(y_{i}\right)$ gives the desired basis of $H^{1}\left(E_{g, n}\right)$.

Corollary 3.4. If $n>1, \alpha\left(E_{g, n}\right)=g$.

Proof. Let $p>1$ be a prime which divides $n$. The bilinear map $\mathbf{Z}^{28} \times \mathbf{Z}^{28}$ $\rightarrow \mathbf{Z}_{n}$ given by

$$
H^{1}\left(E_{g, n}\right) \times H^{1}\left(E_{g, n}\right) \stackrel{\cup}{\rightarrow} \mathbf{Z}_{n} \subset H^{2}\left(E_{g, n}\right),
$$

when tensored with $\mathbf{Z}_{p}$, gives a bilinear map $\mathbf{Z}_{p}^{2 g} \times \mathbf{Z}_{p}^{2 g} \rightarrow \mathbf{Z}_{p}$. This is a bilinear form on the $\mathbf{Z}_{p}$-vector space $\mathbf{Z}_{p}^{2 g}$ whose matrix, relative to the basis $x_{1}, \ldots, x_{2 g}$, is

$$
J=\left[\begin{array}{cc}
0 & I_{g} \\
-I_{g} & 0
\end{array}\right]
$$

If $z_{1}, \ldots, z_{2 g}$ is another basis of $H^{1}\left(E_{g, n}\right)$ and if $z_{i} \cup z_{j}=0,1<i$, $j<g+1$, then the matrix of the form becomes

$$
P^{*} J P=\left[\begin{array}{cc}
0_{g+1} & * \\
* & *
\end{array}\right]
$$

where $P$ is the nonsingular matrix corresponding to the change of basis. But $J$ is nonsingular while the above matrix is clearly singular, so we have reached a contradiction.

The manifolds $E_{g, 0}=S^{1} \times T_{g}$ must be handled a bit differently. We need the following lemma which is easily checked via the Künneth theorem.

LEMMA 3.5. $H^{1}\left(E_{g, 0}\right)=\mathrm{Z}^{28+1}=H^{2}\left(E_{g, 0}\right)$ and there is a basis $x_{1}, \ldots, x_{2 g}, y$ of $H^{1}\left(E_{8,0}\right)$ such that

(1) $x_{i} \cup x_{g+i}=x_{j} \cup x_{g+j}=z, 1<i, j<g$,

(2) $x_{1} \cup y, x_{2} \cup y, \ldots, x_{2 g} \cup y, z$ form a basis of $H^{2}\left(E_{8,0}\right)$,

(3) $x_{i} \cup x_{j}=0,|i-j| \neq g$. 
In particular, of course, $\alpha\left(E_{g, 0}\right)>g$. We must prove the reverse inequality for all $g>1$.

Let $V \subset H^{1}\left(E_{g, 0}, Q\right)$ be the vector subspace spanned by $\left\{x_{1}, \ldots, x_{2 g}\right\}$. Define the bilinear form $\varphi: V \times V \rightarrow \mathbf{Q}$ by

$$
\varphi(v, w) \cdot z=v \cup w .
$$

Then, (3.5) implies that the matrix of $\varphi$ relative to the basis $\left\{x_{1}, \ldots, x_{2 g}\right\}$ is

$$
\Phi=\left[\begin{array}{cc}
0 & I_{g} \\
-I_{g} & 0
\end{array}\right]
$$

This matrix is nonsingular. As in the proof of (3.4), the following will be enough to prove $\alpha\left(E_{g, 0}\right)<g$.

Lemma 3.6. If $\alpha\left(E_{g, 0}\right)>g$, there is a basis $\beta_{1}, \ldots, \beta_{2 g}$ of $V$ such that $\varphi\left(\beta_{i}\right.$, $\left.\beta_{j}\right)=0,1<i, j<g+1$.

Proof. There will be linearly independent elements $z_{1}, \ldots, z_{g+1}$ of $H^{1}\left(E_{g, 0} ; \mathrm{Q}\right)$ with $z_{i} \cup z_{j}=0,1<i, j<g+1$. Express these elements in terms of the basis given in (3.5) as follows:

Then

$$
z_{i}=\sum_{j=1}^{2 g} a_{j}^{i} x_{j}+a_{2 g+1}^{i} y .
$$

$$
0=z_{i} \cup z_{k}=\left(\sum_{j=1}^{g} a_{j}^{i} a_{g+j}^{k}-a_{g+j}^{i} a_{j}^{k}\right) z+\sum_{j=1}^{2 g}\left(a_{j}^{i} a_{2 g+1}^{k}-a_{j}^{k} a_{2 g+1}^{i}\right) x_{j} \cup y
$$

and from (3.5) we can conclude $0=\sum_{j=1}^{g}\left(a_{j}^{i} a_{g+j}^{k}-a_{g+j}^{i} a_{j}^{k}\right)$ and so, if we set

$$
\beta_{i}=z_{i}-a_{2 g+1}^{i} y=\sum_{j=1}^{2 g} a_{j}^{i} x_{j}, \quad 1 \leqslant i \leqslant g+1,
$$

we obtain $\beta_{i} \cup \beta_{k}=0,1 \leqslant i, k \leqslant g+1$. In order to show that $\left\{\beta_{1}, \ldots, \beta_{g+1}\right\}$ is linearly independent, it will be enough to show that $y$ is linearly independent of $\left\{z_{1}, \ldots, z_{g+1}\right\}$. This will complete the proof.

Suppose $y=\sum_{i=1}^{g+1} b_{i} z_{i}$. Then

$$
0=\left(\sum b_{i} z_{i}\right) \cup z_{k}=y \cup z_{k}=\sum_{j=1}^{2 g} a_{j}^{k} y \cup x_{j}, \quad 1<k<g+1 .
$$

By (3.5) we conclude that all $a_{j}^{k}=0$ for $j<2 g$, so $z_{k}=a_{2 g+1}^{k} y, 1<k<g$ +1 . Since we are assuming $g \geqslant 1$, this contradicts the linear independence of $\left\{z_{1}, \ldots, z_{g+1}\right\}$.

Formula (3.1) is now completely proven. It is natural to ask whether Theorem A gives the best upper bound for $\gamma(F)$ on $E_{g, n}$. For $n \neq 1$, it is easy to show that this is true. 
THEOREM 3.7. If $n \neq 1$ and $(n, g) \neq(0,0)$, then there is a foliation $F$ of $E_{g, n}$ with $\gamma(F)=\varphi(g)$.

Proof. The surface $T_{g}$ supports a set of disjointly imbedded circles $\sigma_{1}, \ldots, \sigma_{g}$ which together do not disconnect $T_{g}$. Thus $\left\{\pi^{-1}\left(\sigma_{1}\right), \ldots, \pi^{-1}\left(\sigma_{g}\right)\right\}$ is an admissible system in $E_{g, n}$ of rank $g$. By (2.2), there is a foliation $F$ with $\gamma(F) \geqslant \varphi(g)$. If $n \neq 1$ and $(n, g) \neq(0,0)$, (3.1) says that $\alpha\left(E_{g, n}\right)=g$; hence Theorem A says that $\gamma(F)<\varphi(g)$.

We do not know whether $E_{g, 1}$ admits a foliation $F$ with $\gamma(F)=\varphi(2 g)$, but we doubt it.

\section{REFERENCES}

1. L. Conlon and S. Goodman, Opening closed leaves in foliated 3-manifolds, Topology 14 (1975), 59-61.

2. S. Goodman, Closed leaves in foliated 3-manifolds, Thesis, Saint Louis Univ., 1974.

3. S. P. Novikov, Topology of foliations, Trudy Moskov. Mat. Obšč. 14 (1965), 248-278; English transl., Trans. Moscow Math. Soc. 14 (1967), 268-304. MR 34 \#824.

4. C. C. Pugh, A generalized Poincaré index formula, Topology 7 (1968), 217-226. MR 37 \#4828.

5. P. A. Schweitzer, Codimension one plane fields and foliations, Differential Geometry (Proc. Sympos. Pure Math., vol. 27, part 1), Amer. Math. Soc., Providence, R. I., 1975, pp. 311-312.

6. W. Thurston, Existence of codimension one foliations (preprint).

7. J. W. Wood, Foliations on 3-manifolds, Ann. of Math. (2) 89 (1969), 336-358. MR 40 \#2123.

Department of Mathematics, Washington University, St. Louis, Missouri 63130

Department of Mathematics, University of North Carolina, Chapel Hill, North Carolina 27514 (Current address of Sue Goodman)

Current address (Lawrence Conlon): Department of Mathematics, Fordham University, New York, New York 10458 